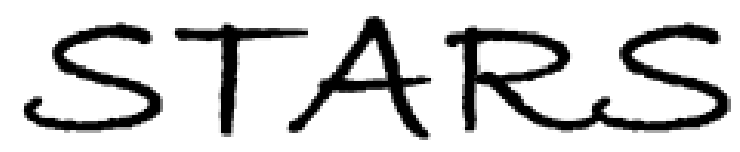

University of Central Florida

STARS

$1-1-2009$

\title{
Titanium Plasma Spectroscopy Studies under Double Pulse Laser Excitation
}

\author{
A. A. I. Khalil \\ M. Richardson \\ University of Central Florida \\ L. Johnson \\ M. A. Gondal
}

Find similar works at: https://stars.library.ucf.edu/facultybib2000

University of Central Florida Libraries http://library.ucf.edu

This Article is brought to you for free and open access by the Faculty Bibliography at STARS. It has been accepted for inclusion in Faculty Bibliography 2000 s by an authorized administrator of STARS. For more information, please contactSTARS@ucf.edu.

\section{Recommended Citation}

Khalil, A. A. I.; Richardson, M.; Johnson, L.; and Gondal, M. A., "Titanium Plasma Spectroscopy Studies under Double Pulse Laser Excitation" (2009). Faculty Bibliography 2000s. 1717.

https://stars.library.ucf.edu/facultybib2000/1717

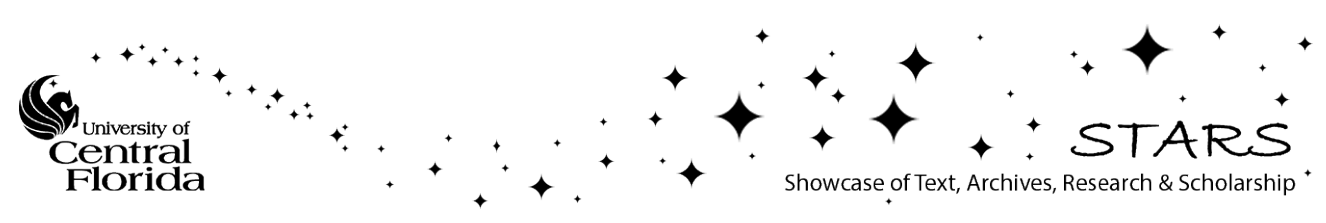




\title{
Titanium Plasma Spectroscopy Studies under Double Pulse Laser Excitation'
}

\author{
A. A. I. Khalil ${ }^{a, b}$, , M. Richardson ${ }^{c}$, L. Johnson ${ }^{d}$, and M. A. Gondal ${ }^{e}$ *** \\ ${ }^{a}$ National Institute of Laser Enhanced Sciences (NILES), \\ Cairo University, Giza, 12613 Egypt \\ ${ }^{b}$ Department of Physics, Faculty of Science for Girls, King Faisal University, \\ 838, Postal Code 31113, Dammam, Saudi Arabia \\ ${ }^{c}$ Laser Plasma Laboratory, College of Optics and Photonics, University of Central Florida, \\ Orlando, FL, 32281 USA \\ ${ }^{d}$ Physics Department, Florida A and M University, Tallahassee, FL, 32307 USA \\ e Physics Department, King Fahd University of Petroleum and Minerals \\ Box 5047, Dhahran, 31261 Saudi Arabia \\ *e-mail: Ahmedasaad68@yahoo.com \\ **e-mail:magondal@kfupm.edu.sa
}

Received April 3, 2009; in final form, April 6, 2009

\begin{abstract}
Laser-induced breakdown spectroscopy (LIBS) was applied for parametric studies of titanium (Ti) plasma using single and double pulsed laser excitation scheme. Here a pulsed Nd:YAG laser was employed for generation of laser produced plasma from solid Ti target at ambient pressure. Several ionized titanium lines were recorded in the 312-334 $\mathrm{nm}$ UV region. The temporal evolution of plasma parameters such as excitation temperature and electron number density was evaluated. The effect of incident laser irradiance, position of the laser beam focal point with respect to the surface of illumination, single and double laser pulse effect on plasma parameters were also investigated. This study contributes to a better understanding of the LIBS plasma dynamics of the double laser pulse effect on the temporal evolution of various Ti emission lines, the detection sensitivity and the optimal dynamics of plasma for ionized states of Ti. The results demonstrate a faster decay of the continuum and spectral lines and a shorter plasma life time for the double pulse excitation scheme as compared with single laser pulse excitation. For double pulse excitation technique, the emissions of Ti lines intensities are enhanced by a factor of five which could help in the improvement of analytical performance of LIBS technique. In addition, this study proved that to avoid inhomogeneous effects in the laser produced plasma under high laser intensities, short delay times between the incident laser pulse and ICCD gate are required.
\end{abstract}

PACS numbers: 29.25.Rm, 29.25.Ni, 41.75.Ak, 42.55.Mv, 42.55.Rz, 42.60.Py, 42.62.Fi

DOI: $10.1134 / \mathrm{S} 1054660 \mathrm{X} 09190116$

\section{INTRODUCTION}

Recently LIBS technique has become very important due to many applications like rapid spectrochemical analysis of materials, technological advances in industrial applications, material processing, medical diagnostics, analytical chemistry, defense and in the other scientific research activities. Ti has many interesting properties, such as biocompatibility, hardness, high corrosion resistance, and light weight and due to these properties it is being applied in aerospace, orthopedic applications, chemical industries and in the vacuum industry [1-6]. In addition, it has the characteristic of low gas release due to protection of the surface with a stable oxide layer. Ti compounds include many stable compounds. For example, $\mathrm{TiO}_{2}$ has chemical stability, a high melting point, and other optical properties that it is widely used in many catalytic and photo-oxidation processes. Ti shows excel-

\footnotetext{
${ }^{1}$ The article is published in the original.
}

lent characteristics of high hardness and wear resistance to many materials. There are some isolated reports on the plasma related studies regarding these compounds under the ambient atmospheric conditions [7-9]. Recently a chemical model based on collisional-radiative transfer for Ti plasma coupled with a fluid dynamic has been suggested $[10,11]$. Keeping in view of the importance of the Ti and its compounds, it is important to improve analytical techniques and to develop new approaches for rapid, reliable and accurate analysis which could allow real-time monitoring of Ti production. It is well known that the analysis of the emitted lines allows the direct atomic composition of the substance under study by using optical techniques and spectral analysis through which one can detect the trace elements within short period of time.

In this paper, we report experiments to understand the mechanism of the single and double pulse excitation using LIBS technique to study the influence of different parameters on spectroscopic temporal evolu- 
tion of various ionic lines of $\mathrm{Ti}$ at ambient pressure. Different parameters studied in this paper include the position of the laser beam focal point with respect to the surface of illumination, incident laser pulse energy and the time interval between laser pulse and opening of ICCD gate. The spectral range recorded simultaneously with this setup covered the ultraviolet (UV) spectral regime $(312-334 \mathrm{~nm})$. The effect of these parameters on line emission intensity enhancement due to the single pulse (SP) and double pulse (DP) excitation and plasma plume life time was investigated by using time resolved LIBS technique.

\section{EXPERIMENTAL SET-UP}

A schematic diagram of the double pulse LIBS system applied in this study is depicted in Fig. 1. Two Qswitched Nd:YAG lasers system $(10 \mathrm{~Hz}, 8 \mathrm{~ns}$, I and II, Model Ultra CFR, Big Sky Laser) [12] were installed in a collinear configuration. The laser beams from these two lasers were guided with help of a dichroic mirror and a polarizer to a test sample at ambient pressure. The wavelength of the first and second lasers was fixed at $532 \mathrm{~nm}$. Both laser beams were focused onto the sample with an A/R-coated fused silica planoconvex lenses $(f=200 \mathrm{~mm})$. To produce a more defined focus, both beams were expanded by a ratio of $3: 1$ by using a simple Galilean telescope before the irradiation on the target. A delay generator (Stanford Research System, model DG535) was applied for controlling the time sequence and synchronization of the both lasers. The delay time between two laser beams was fixed $=0.5 \mu$ s during whole experiment. Here, the Independent firing of the two laser pulses was achieved by an external delay generator. The time jitter between the firing of two lasers was $<10 \mathrm{~ns}$. A polished Ti target was placed on a rotary stage to provide a fresh surface for incident laser radiation to avoid the crust formation. The effect of position of the laser beam focal point with respect to the illumination surface $(2.45 \mathrm{~mm}$ in front of the surface, on the surface, $1.7 \mathrm{~mm}$ behind the surface and $4.7 \mathrm{~mm}$ behind the surface) was studied.

Two A/R-coated fused silica plano-convex lenses $(f=75 \mathrm{~mm})$ were used to image the laser-induced plasma spectrum onto the fiber bundle. The other end of the fiber bundle illuminates the entrance slit of a $30 \mathrm{~cm}, \mathrm{f} / 4$-aperture spectrometer (Acton Research SpectraPro 300i) [13] equipped with a 2400 groove $/ \mathrm{mm}$ holographic grating blazed at $500 \mathrm{~nm}$ to record the Ti plasma spectra in the UV and visible region. The fiber bundle is $1 \mathrm{~m}$ long and consists of 19-200 $\mu \mathrm{m}$ diameter fibers arranged in a single column at one end. Spectral emission was detected by a gated Intensified Charge Coupled Device system (ICCD) $690 \times 128$ pixels (Andor Technologies, DH501-18F-XX). The ICCD gate delay (time between laser excitation and opening of camera shut- ter for plasma emission recording) was varied to investigate the temporal evolution of the plasma, while the gate width was kept constant at $50 \mathrm{~ns}$.

The Ti targets was in square shape having dimensions $=5 \times 5 \times 9 \mathrm{~cm}^{3}$. Time dependence of several Ti ionic emission lines for SP-mode and DP-mode respectively at ambient pressure was studied. All the spectral transitions of the investigated ionic emission lines of Ti were identified using the NIST spectroscopic database [14].

\section{RESULTS AND DISCUSSION}

In this study, the dependence of temporal evolution of various plasma parameters was carried out by recording the ionic transitions in Ti by using LIBS technique. Figures 2a, $2 b$ show typical emission spectra of the plasma produced by laser ablation of Ti target in the UV region for single pulse (SP) and double pulse (DP) modes. It is noticed that the intensity of Ti spectra increased by a factor of five in a DP-mode if $2 \times$ $20 \mathrm{~mJ}$ is applied instead of in a SP-mode of $40 \mathrm{~mJ}$ pulse. The various spectral lines of Ti were identified using NIST data base and are indicated in Fig. 2a. It is worth mentioning that the Single and Double Pulse laser induced plasma studies of $\mathrm{Ti}$ in the UV region (312-334) nm at ambient pressure using LIBS technique are reported for the first time.

Figure 3 depicts the spectral emission from SP- and DP-LIBS recorded at different positions of the laser focal distance relative to the target surface (for SP, Figs. 3a, 3c, 3e, 3g; where focal length $f=2.45 \mathrm{~mm}$ in front of the surface, on the surface and $f=1.7$ and $4.7 \mathrm{~mm}$ behind the surface and for DP, Figs. 3b, 3d, 3f, $3 \mathrm{~h}$ where $f=2.45 \mathrm{~mm}$ in front of the surface, on the surface and $f=1.7$ and $4.7 \mathrm{~mm}$ behind the surface, respectively at ambient pressure. The spectra are collected at different time interval delays between laser pulse and ICCD gate delay ranging from 0 to $6000 \mathrm{~ns}$. Each fragment of spectra in Fig. 3 belongs to a different portion of the LIBS temporal distribution. The continuum emission intensity in the plasma around $324 \mathrm{~nm}$ is greatest in the region close to the Ti target surface. This continuum arises around the 0 delay due to free-free Bremsstrahlung and electron-ion recombination [15-18]. At further distances away from the target, the spectra are dominated by low lying charged ionic states (Ti I-II) as shown in Figs. $3 \mathrm{a}-3 \mathrm{~h}$. The emission lines become progressively narrower as a consequence of the electron number density distribution. The excitation temperature decreases at longer times as a result of plasma cooling. The maximum intensity of the spectral lines is reached after a certain time which is characteristic of ionic specie depending on the observation location, which illustrates the most populated section of LIBS. For the higher degree of ionization, it can be seen that in most part of the spectra, the ionic lines are proportionally more intense than those of the atoms. The temporal distribution of 

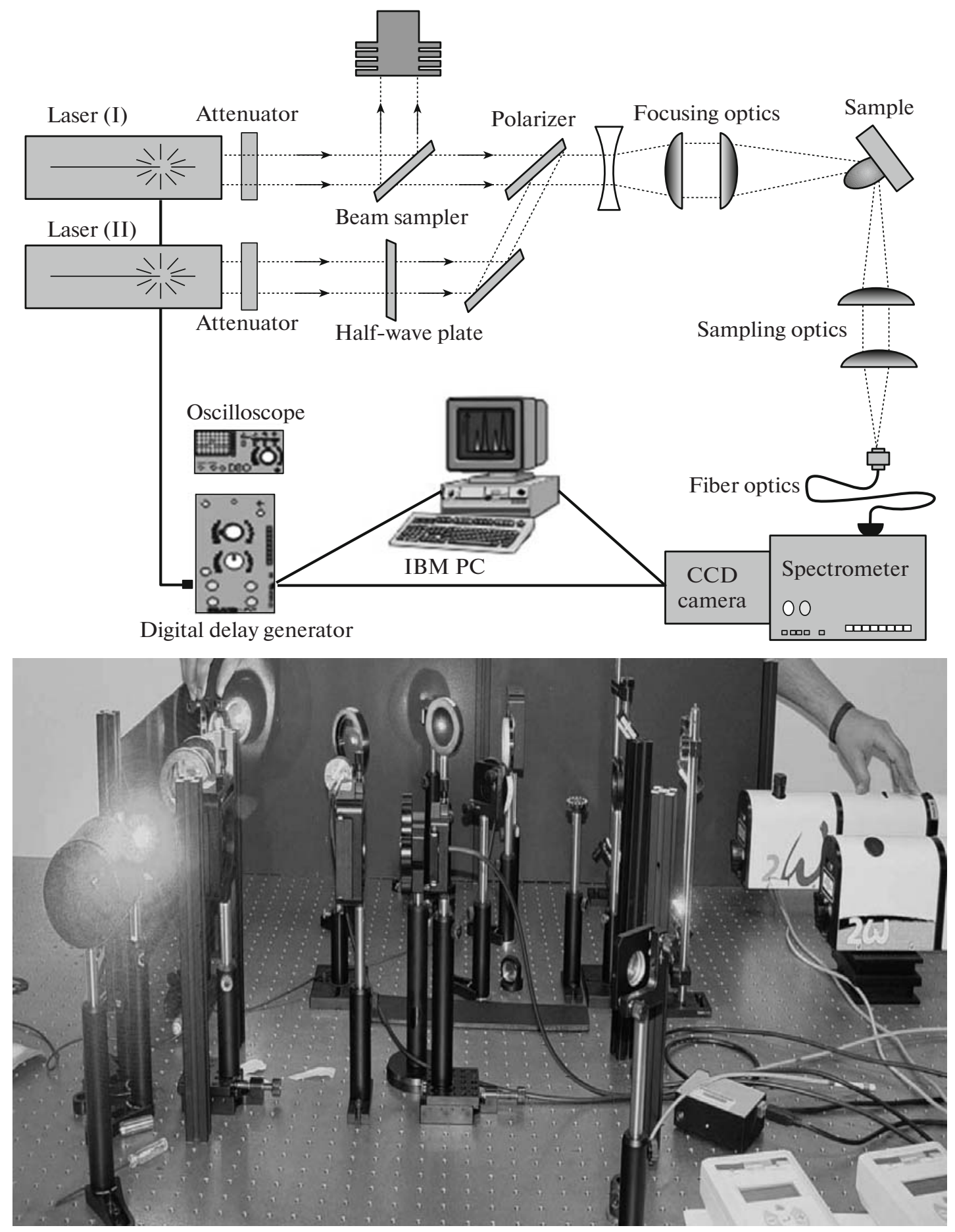

Fig. 1. Schematic diagram of the experimental setup.

the LIBS line intensities on the tails, which corresponds to the colder part of the plasma, it is possible to observe the disappearance of ionic lines. This phenomenon has taken place for SP- and DP-modes at different laser focusing positions at front, on and behind the surface as shown in Fig. 3. Here the time resolved spectra are recorded for different time delays between the laser pulse and the triggering of the ICCD camera. The gate width of the ICCD camera was $50 \mathrm{~ns}$ and laser irradiance was $1.35 \times 10^{10} \mathrm{~W} \mathrm{~cm}^{-2}$. 

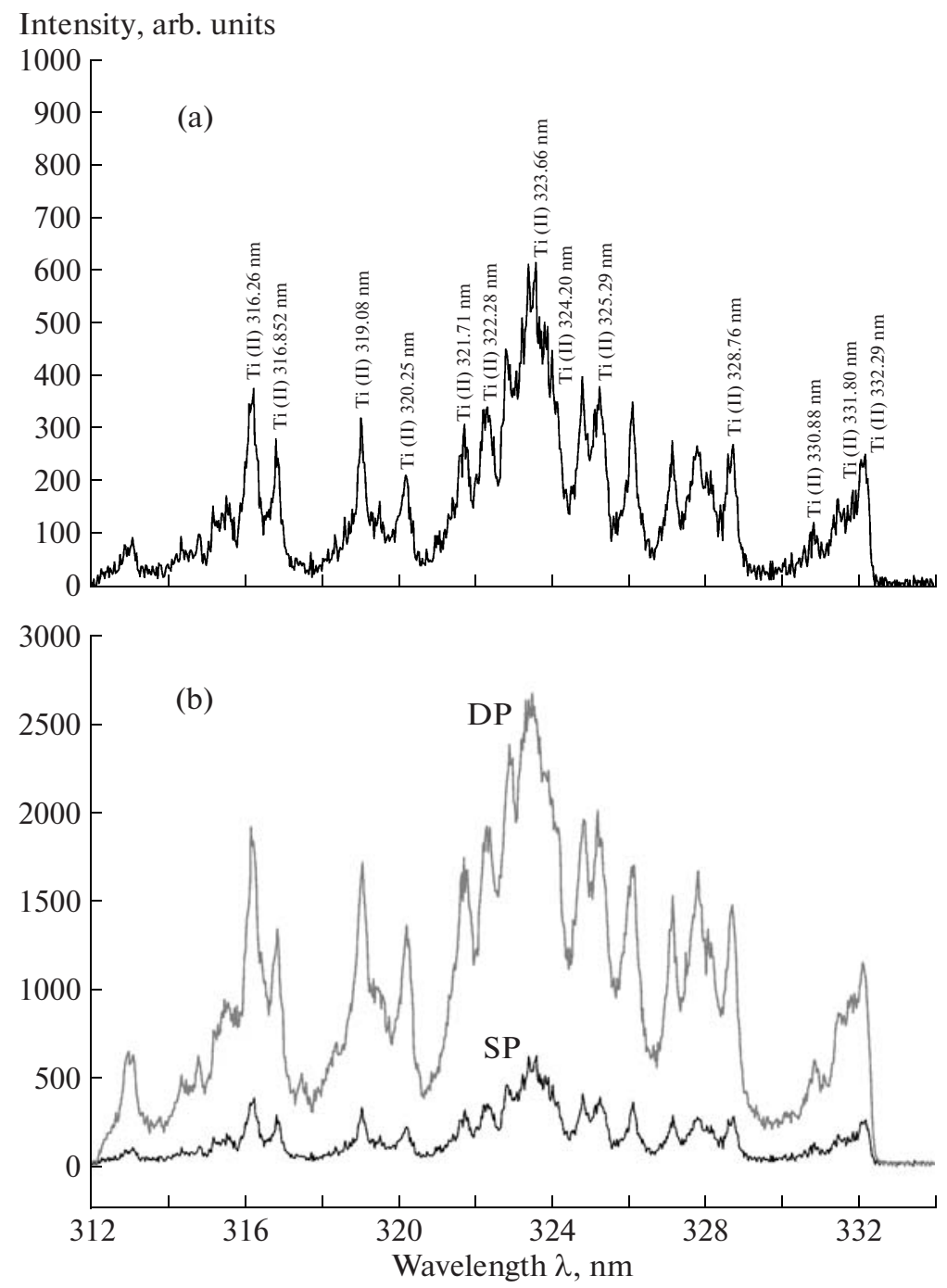

Fig. 2. Typical spectra recorded for both (a) single pulse (SP) and (b) double pulse (DP) by focusing the laser direct on the Ti target surface.

Figure 4 depicts the time dependence of the emission intensities of several $\mathrm{Ti}$ ionic lines at $\mathrm{Ti}$ II (316.26), Ti II (321.71), Ti II (323.66), Ti II (325.29), and Ti II (332.29) $\mathrm{nm}$ for different single and double (a, b, c, d, e) pulses, respectively at ambient pressure. Figure 4 depicts that the emission intensity of all ionized Ti lines which are sharply decreased at the early stage of plasma in SP-mode and slightly decreased at the longer time delays. This phenomenon clearly took place when the laser focused at $2.54 \mathrm{~mm}$ in front of the target, on the target and $1.7 \mathrm{~mm}$ behind the target surface except at $4.7 \mathrm{~mm}$ behind the surface, the intensity of all emissions lines became nearly equal or slightly changed with time. In Fig. 4, it can be seen that the emission intensity of all ionized Ti lines in DP-mode increased initially as the gate delay changed from 0 to $500 \mathrm{~ns}$, having a peak at $500 \mathrm{~ns}$ and started to decay monotonically for gate delays beyond $500 \mathrm{~ns}$ at differ- ent positions, by focusing the laser at $2.54 \mathrm{~mm}$ front of the target, on the target, 1.7 and $4.7 \mathrm{~mm}$ behind the target surface. The maximum emission intensity of the all Ti ionized lines was obtained at a position when the laser was focused well on the Ti surface as shown in Fig. 5.

Figure 5 depicts the change in emission intensity at different laser focus positions from the Ti surface. The maximum spatial range for the ions is limited by the drop in recorded intensity with position from the surface in SP- and DP-LIBS experiments. It was found that focusing the laser beam on the target surface generates high emission intensity for both SP- and DPmodes of all ionized Ti emission lines. It is well known that, the species with higher degree of ionization have higher velocities because of the Coulomb fields generated by negatively charged electrons escaping from the plasma plume. 

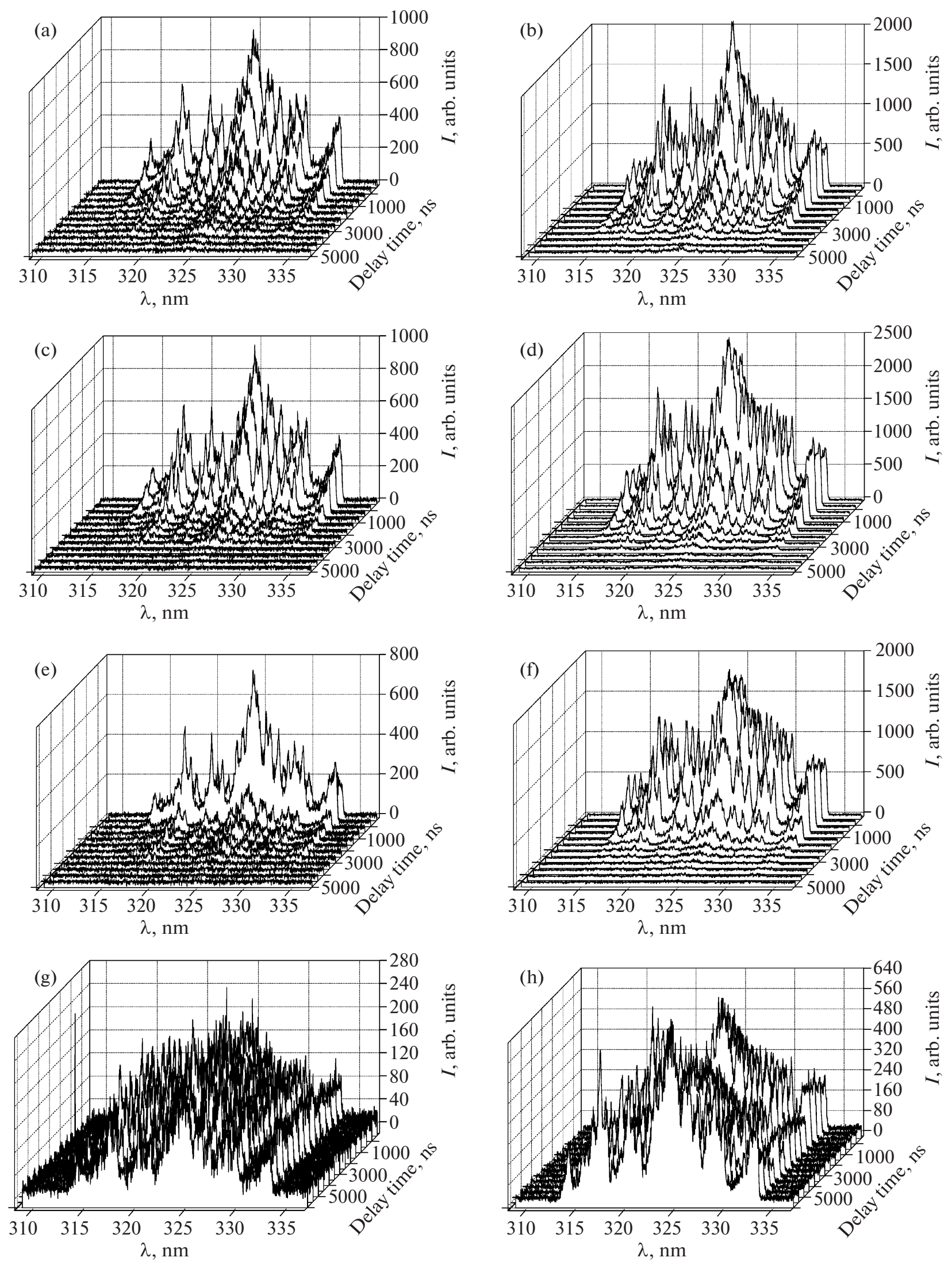

Fig. 3. Typical spectra recorded for SP-mode excitation (a, c, e, g) $2.45 \mathrm{~mm}$ in front of the surface, on the surface, $1.7 \mathrm{~mm}$ behind the surface, $4.7 \mathrm{~mm}$ behind the surface and DP-mode excitation $(\mathrm{b}, \mathrm{d}, \mathrm{f}, \mathrm{h}) 2.45 \mathrm{~mm}$ in front of the surface, on the surface, $1.7 \mathrm{~mm}$ behind the surface, $4.7 \mathrm{~mm}$ behind the surface, respectively at ambient pressure, on Ti target. 

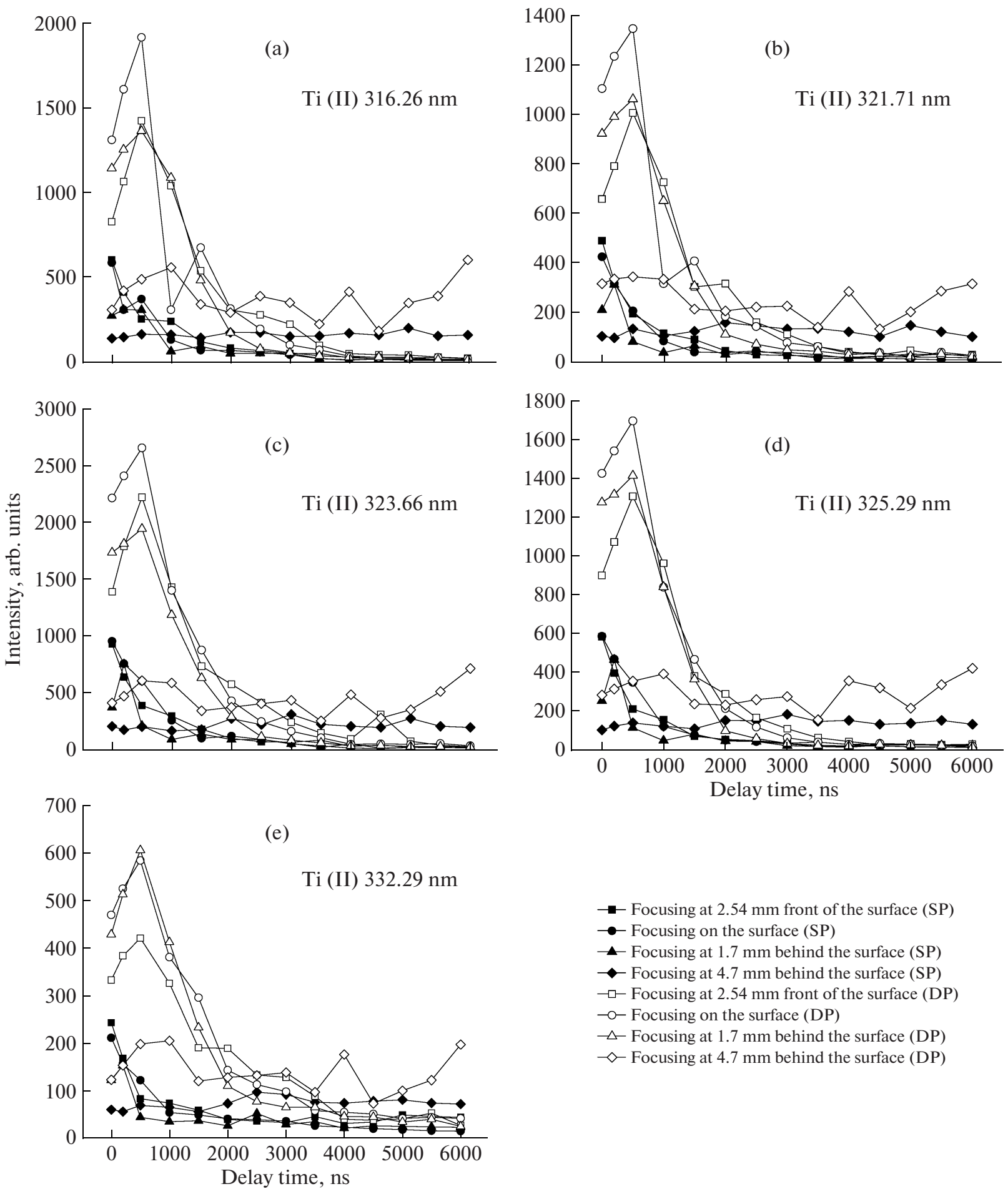

Fig. 4. Time dependence of the emission intensities of several titanium lines at 316.85, 321.42, 324.20, 325.43, and 332.95 nm for different SP- and DP-modes (a, b, c, d, e) at different focusing positions relative to the target surface recorded at ambient pressure.

The temporal evolution of the structures of SP- and DP-LIBS of different ionic states by focusing the laser on the Ti surface was also investigated as shown in
Fig. 6. The emission intensity is different for different ionized Ti lines. The emission intensity is enhanced with increasing degree of ionization at early stages par- 
Intensity, arb. units
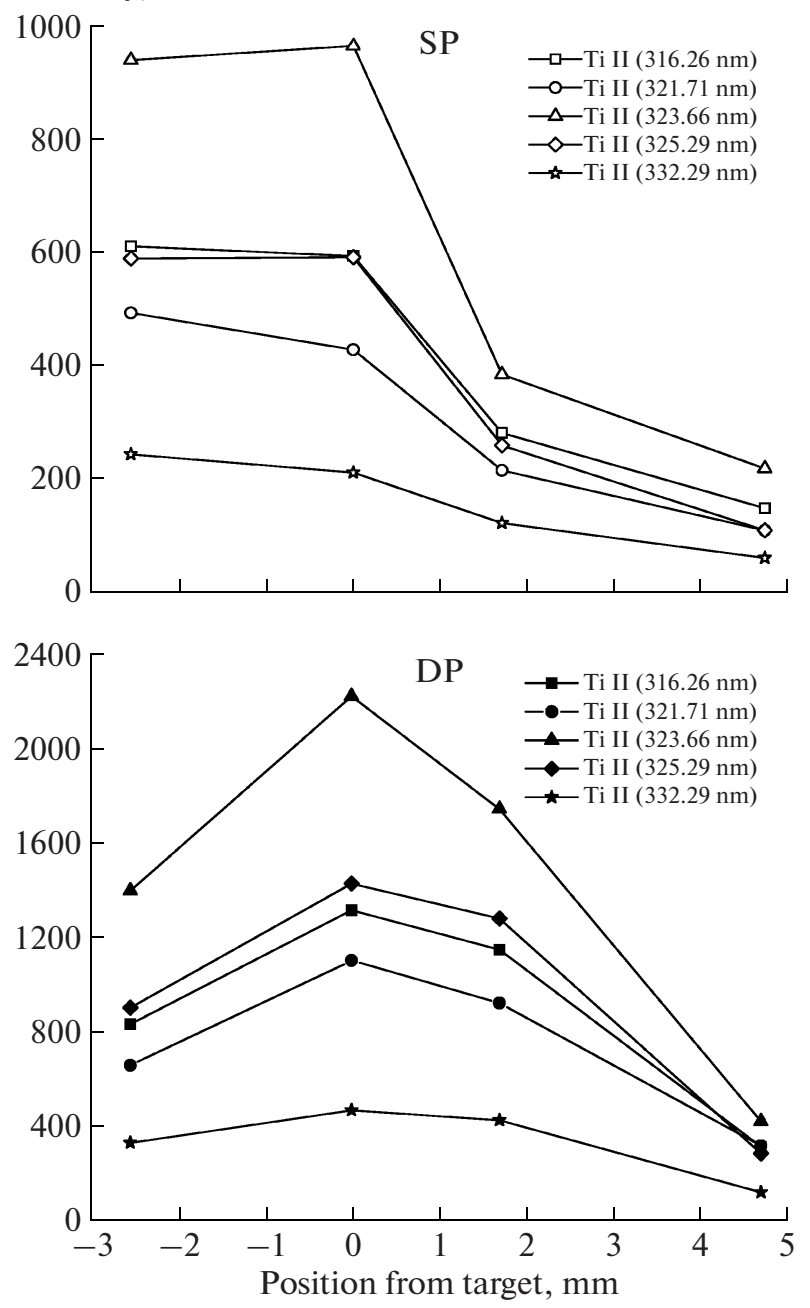

Fig. 5. Emission intensity trends of SP-and DP at different laser focusing positions relative to the Ti target at $2.45 \mathrm{~mm}$ in front of the surface, on the surface, $1.7 \mathrm{~mm}$ behind the surface and $4.7 \mathrm{~mm}$ behind the surface for different ionic species.

ticularly, for Ti II $323.66 \mathrm{~nm}$ in both single and double pulse. In double pulse case, it is obvious that the emissions of all ionic stages are delayed by $500 \mathrm{~ns}$ after the maximum of the laser pulse. Assuming that the maximum emission intensity at a given distance in front of the target corresponds to the time when the plasma front passes through the observation region. The species with a higher degree of ionization have higher velocities because of the coulomb fields generated by negatively charged electrons escaping from the plume. Avalanche ionization due to long wavelength (IR-VIS) laser excitation and multi-photon ionization phenomenon using short wavelength lasers (UV) are responsible for Laser Induced Breakdown and plasma formation. The effect of laser irradiance on the emission intensity from the different ionic lines of Ti target was also carried out in this study. The spectra were
Intensity, arb. units

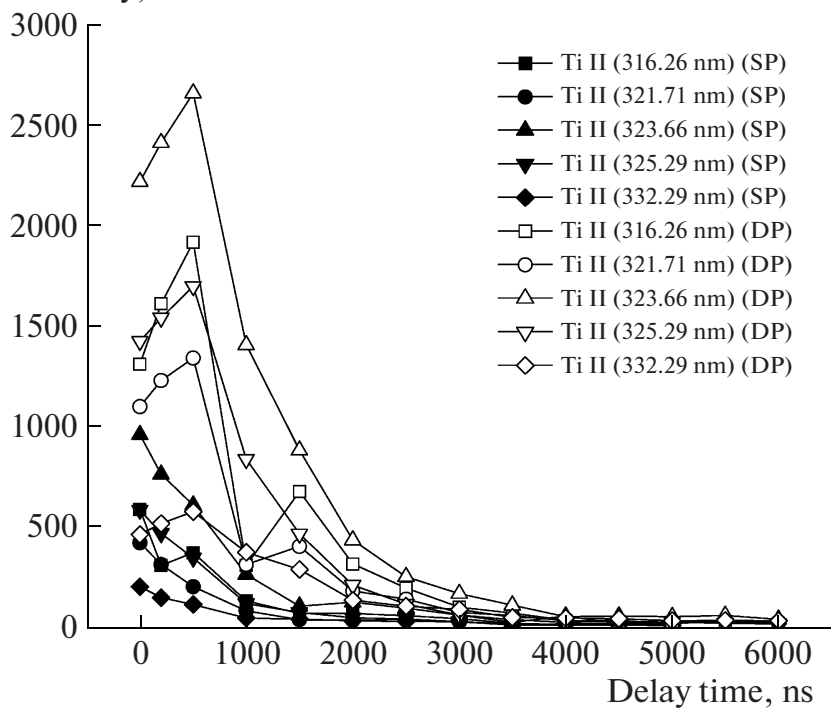

Fig. 6. Emission intensity plotted for SP- and DP-modes of different ionic lines versus time by focusing the laser beam on the target surface.

recorded for the maximum irradiance of the laser pulse, when the laser was focused on the target surface for different laser irradiances and averaged over the 10 measurements under the same laser irradiance. Figure 7 clearly shows that the emission intensity from Ti ions (Ti II (316.26), Ti II (321.71), Ti II (323.66), Ti II (325.29) and Ti II (332.29) nm) initially increases slowly with laser irradiance and then followed on fast scale toward the saturation. This phenomenon could be understood as follows. By increasing the laser irradiance, more excited species like ions and the number of free electrons are generated and due to this, the electron density increases. The plasma temperature is lower for lower laser irradiance at a fixed delay time. The laser irradiance was varied between 0.34 and $2.05 \mathrm{~W} \mathrm{~cm}^{-2}$ and for each laser irradiance, averaged spectra was recorded. The error bars indicate the standard deviation of 10 LIBS signals. Due to the rough surface, the error bar is up to $10 \%$. At higher laser irradiance, saturation has been appeared in the region of laser irradiance $\left[(1.35-2.05) \times 10^{10} \mathrm{~W} \mathrm{~cm}^{-2}\right]$ for all emission lines. The saturation in the emission intensity starts near the laser intensity $1.36 \times 10^{10} \mathrm{~W} \mathrm{~cm}^{-2}$. This saturation in the emission intensity at higher laser irradiance is probably due to plasma shielding i.e. self absorption and/or reflection of the laser photons by the plasma itself. This could be due the increase of plasma species (atoms, electrons and ions), that are generated due to higher ablation rate of the Ti target at higher laser energy [19-26]. These species can interact with incoming laser photons, gaining more heat and ionization and resulting in an increase in absorption of the laser energy. The plasma behavior seems to 


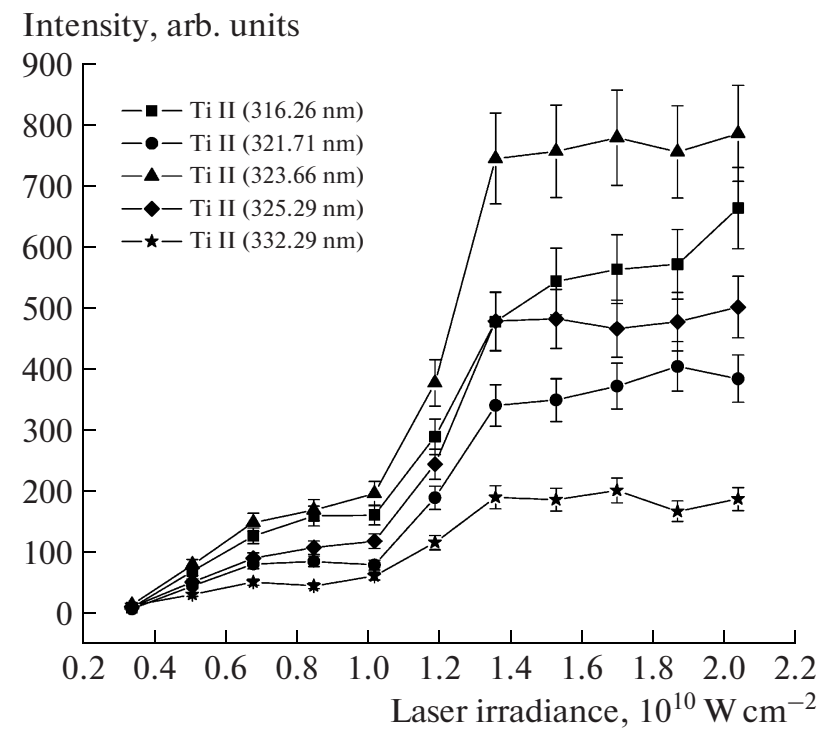

Fig. 7. Dependence of emission intensity on the SP laser irradiance for different ionic transitions of Ti by focusing the laser beam on the target surface.

be an optically thick and effectively shields the target surface from the part of the laser pulse and a self-regulating regime may exist. Due to random collisions with plasma species, the electrons gain energy from the oscillation field. Therefore, the ionization by electron impact and formation of dense plasma by photoionization leads to an increase in the electron energy. The energy of a free electron oscillating in an electromagnetic field is given by [27]

$$
U_{\mathrm{os}}=\frac{e^{2} E^{2}}{2 m \omega^{2}},
$$

where $\omega$ is the angular frequency of the laser field, $e$ is the electron charge, and $m$ is the mass of the free electron. Under our experimental conditions, the calculated $U_{\text {os }}$ is about $10^{-4} \mathrm{eV}$ which seems to be low. Although hot electrons with much higher kinetic energies can be generated by the resonance absorption of laser radiation, and this phenomenon take place only when the laser beam is not incident normally on the target [27] as in our experiment. The resonance absorption can not be taken into consideration in our case as the resonance absorption phenomena in laser produced plasmas take place at high plasma density. Usually it takes place at high laser intensities of about $10^{14} \mathrm{~W} \mathrm{~cm}^{-2}$ or higher [28] in comparison with our investigation where the laser intensity of $10^{10} \mathrm{~W} \mathrm{~cm}^{-2}$ was applied. The other processes such as Photo-ionization, thermal and impact ionizations may affect the absorption coefficient of the plasma. In our case of $\mathrm{Ti}$ plasma produced by Nd:YAG laser ( $\mathrm{SHG}$ ), an inverse Bremsstrahlung could be the dominant mechanism for plasma absorption at these laser irradiance region. The inverse Bremsstrahlung process due to electron-ion scattering is usually described by the inverse absorption length, as [29],

$$
\sigma_{\mathrm{ib}} \approx 1.27 \times 10^{-46} \lambda^{3} Z^{2} n_{e} n_{i} T_{e}^{-1 / 2}\left[1-\exp \left(-\frac{h v}{T_{e}}\right)\right],
$$

where $\lambda$ is the wavelength of the laser photons in $\mathrm{nm}$, $T_{e}$ is the electron temperature in $\mathrm{eV}, h v$ is the laser photon energy in $\mathrm{eV}, \mathrm{Z}$ is the ionic charge, and $n_{i}$ and $n_{e}$ are the ion and electron densities, respectively. From Eq. (2), it can be noticed that inverse Bremsstrahlung is an efficient absorption process, in particular at visible and IR wavelengths, because of its wavelength dependence of $\lambda^{3}$.

\subsection{Electron Temperature}

The electron (or plasma) temperature can be determined with the well-known Boltzmann method from measurement of relative line intensities, provided that their transition probabilities $\left(A_{j k}\right)$ from a given excitation state are known. The plasma temperature was estimated from the emission intensity of six ionic $\mathrm{Ti}$ lines recorded from the laser induced plasma. These lines are well resolved, with well-known transition probabilities. This condition is valid for the plasma being assumed in state of Local Thermodynamic Equilibrium (LTE) and optically thin for the lines observed. Therefore, the populations of the excited states follow a Boltzmann distribution, and their relative emissivity $\left(\varepsilon_{j k}\right)$ can be given by [30-33],

$$
\ln \left(\frac{\lambda_{j k} \varepsilon_{j k}}{A_{j k} g_{j}}\right)=-\frac{E_{j}}{k T_{\text {exc }}}+\ln \left(\frac{N(T)}{Q(T)}\right),
$$

where $\varepsilon_{j k}, A_{j k}, \lambda_{j k}$, and $g_{j}$ are the relative emissivity, the transition probability, wavelength and the statistical weight for the upper level, respectively; $E_{j}$ is the excited level energy; $T_{\text {exc }}$ is the excitation temperature; $k$ and $h$ are the Boltzmann and Planck constants, respectively; $Q(T)$ is the partition function and $N(T)$ the atomic (or ion) density. The wavelengths, degeneracy, the transition probability, statistical weight, and excitation energy of these Ti lines are listed in table and were taken from NIST atomic spectra database [14]. By using the equation 3, the plasma temperature can be calculated from the slop of the line drawn by plotting $\ln \left(\frac{\lambda_{j k} \varepsilon_{j k}}{A_{j k} g_{j}}\right)$ versus the energy $\left(E_{j}\right)$ as shown in the inset in Fig. 8a.

Figures $8 \mathrm{a}, 8 \mathrm{~b}$ depict the temporal evolution of the calculated excitation temperature for Ti lines recorded with our LIBS setup. The plasma temperature for ionized $\mathrm{Ti}$ lines increases at the early stages from 0 to $500 \mathrm{~ns}$ due to absorption of the laser energy by means of electrons via the inverse Bremsstrahlung absorption process. After a delay time $=500 \mathrm{~ns}$, a decay in elec- 
Spectroscopic data for various Ti ionic lines investigated in this work and used for the calculations of plasma parameters (electron temperature and density)

\begin{tabular}{l|c|c|c|c|c|c}
\hline $\begin{array}{c}\text { Wavelength } \\
\lambda_{j k}, \mathrm{~nm}\end{array}$ & $\begin{array}{c}\text { Transition } \\
\text { probability } A_{j k}, \mathrm{~s}^{-1}\end{array}$ & $\begin{array}{c}\text { Lower energy } \\
\text { level } E_{i}, \mathrm{~cm}^{-1}\end{array}$ & $\begin{array}{c}\text { Upper energy } \\
\text { level } E_{k}, \mathrm{~cm}^{-1}\end{array}$ & Transition & $\begin{array}{c}\text { Statistical } \\
\text { weight } g_{i}\end{array}$ & $\begin{array}{c}\text { Statistical } \\
\text { weight } g_{k}\end{array}$ \\
\hline 316.26 & $3.9 \times 10^{7}$ & 1087.32 & 32697.99 & $3 d^{3} \longrightarrow 3 d^{2}\left({ }^{3} F\right) 4 p$ & 8 & 6 \\
316.85 & $4.1 \times 10^{7}$ & 1215.84 & 32767.07 & $3 d^{3} \longrightarrow 3 d^{2}\left({ }^{3} F\right) 4 p$ & 10 & 8 \\
319.087 & $1.3 \times 10^{8}$ & 8744.25 & 40074.52 & $3 d^{2}\left({ }^{1} D\right) 4 s \longrightarrow 3 d^{2}\left({ }^{1} D\right) 4 p$ & 6 & 8 \\
319.99 & $9.4 \times 10^{7}$ & 386.874 & 31628.668 & $3 d^{2} 4 s^{2} \longrightarrow 3 d^{3}\left({ }^{4} F\right) 4 p$ & 9 & 11 \\
320.25 & $1.1 \times 10^{8}$ & 8710.44 & 39926.66 & $3 d^{2}\left({ }^{1} D\right) 4 s \longrightarrow 3 d^{2}\left({ }^{1} D\right) 4 p$ & 4 & 6 \\
321.42 & $6.5 \times 10^{6}$ & 386.874 & 31489.451 & $3 d^{2} 4 s^{2} \longrightarrow 3 d^{3}\left({ }^{4} F\right) 4 p$ & 9 & 9 \\
321.706 & $1.69 \times 10^{7}$ & 225.73 & 31301.01 & $3 d^{2}\left({ }^{3} F\right) 4 s \longrightarrow 3 d^{2}\left({ }^{3} F\right) 4 p$ & 8 & 10 \\
322.28 & $2.6 \times 10^{7}$ & 94.10 & 31113.65 & $3 d^{2}\left({ }^{3} F\right) 4 s \longrightarrow 3 d^{2}\left({ }^{3} F\right) 4 p$ & 6 & 8 \\
323.66 & $1.11 \times 10^{8}$ & 225.73 & 31113.65 & $3 d^{2}\left({ }^{3} F\right) 4 s \longrightarrow 3 d^{2}\left({ }^{3} F\right) 4 p$ & 8 & 8 \\
324.20 & $1.16 \times 10^{8}$ & 0 & 30836.32 & $3 d^{2}\left({ }^{3} F\right) 4 s \longrightarrow 3 d^{2}\left({ }^{3} F\right) 4 p$ & 4 & 4 \\
325.291 & $3.9 \times 10^{7}$ & 225.73 & 30958.50 & $3 d^{2}\left({ }^{3} F\right) 4 s \longrightarrow 3 d^{2}\left({ }^{3} F\right) 4 p$ & 8 & 6 \\
325.43 & $2.0 \times 10^{7}$ & 393.44 & 31113.65 & $3 d^{2}\left({ }^{3} F\right) 4 s \longrightarrow 3 d^{2}\left({ }^{3} F\right) 4 p$ & 10 & 8 \\
328.766 & $1.4 \times 10^{8}$ & 15265.62 & 45673.62 & $3 d^{2}\left({ }^{1} G\right) 4 s \longrightarrow 3 d^{2}\left({ }^{1} G\right) 4 p$ & 8 & 10 \\
330.881 & $4.5 \times 10^{6}$ & 1087.32 & 31301.01 & $3 d^{3} \longrightarrow 3 d^{2}\left({ }^{3} F\right) 4 p$ & 8 & 10 \\
331.802 & $6.0 \times 10^{6}$ & 983.89 & 31113.65 & $3 d^{3} \longrightarrow 3 d^{2}\left({ }^{3} F\right) 4 p$ & 6 & 8 \\
332.294 & $3.96 \times 10^{7}$ & 1215.84 & 31301.01 & $3 d^{3} \longrightarrow 3 d^{2}\left({ }^{3} F\right) 4 p$ & 10 & 10 \\
332.95 & $3.25 \times 10^{7}$ & 1087.32 & 31113.65 & $3 d^{3} \longrightarrow 3 d^{2}\left({ }^{3} F\right) 4 p$ & 8 & 8 \\
333.52 & $2.93 \times 10^{7}$ & 983.89 & 30958.50 & $3 d^{3} \longrightarrow 3 d^{2}\left({ }^{3} F\right) 4 p$ & 6 & 6 \\
\hline
\end{tabular}

tron temperature was noticed for different focus positions at $2.54 \mathrm{~mm}$, on the surface, 1.7 and $4.7 \mathrm{~mm}$ behind the Ti surface for the single pulse excitation respectively. After $6000 \mathrm{~ns}$, the Ti lines finally disappear. Also, it can be observed that in the DP-excitation, electron temperature increases gradually at the early stages in the range from 0 to $500 \mathrm{~ns}$ and slightly decreases after $500-\mathrm{ns}$ for different focus positions at $2.54 \mathrm{~mm}$ in front of the surface, on the surface, 1.7 and $4.7 \mathrm{~mm}$ behind the Ti surface, respectively. As one can notice from Figs. 8a, 8b, the electron temperature varies from 10000 to $28000 \mathrm{~K}$ for single pulse (Fig. 8a) and for double pulse mode it varies from 8000 to $32000 \mathrm{~K}$ for different laser focal point position with respect to the target surface. The statistical error bar for the determination of excitation temperature is approximately less than 5\%. As in the ns regime, a significant fraction of the laser energy is absorbed by the plasma due to inverse Bremsstrahlung so it should increase the plasma temperature as well as the electron density at early stage of plasma generation. It was also observed that the excitation temperature decreased due to the long time delays by varying the laser focal positions away from the Ti surface as shown in Fig. 8. This may be due to the fact that thermal energy is rapidly converted into kinetic energy, by attaining maximum expansion velocities and leads to cooling of the plasma. Furthermore, it can be seen that the maxi- mum excitation temperature is slightly higher in the case of DP-mode than that obtained with SP-mode.

\subsection{Electron Densities}

The spectroscopic technique for determining $N_{e}$ depends upon the measurement of the Stark broadening of some optical lines [34]. Stark broadening of the spectral lines in plasma results from collisions with charged species leading to both a broadening of the line and a shift in the peak wavelength. From Stark broadening, $N_{e}$ can be calculated easily. Electrons in the plasma can perturb the energy levels of the individual atoms which broaden the emission lines originating from these excited levels. The full width at half maximum (FWHM) $\Delta \lambda_{1 / 2}$ of the Stark-broadened line is given by $[35,36]$.

$$
\begin{gathered}
\Delta \lambda_{1 / 2}(A)=2 \omega\left(\frac{N_{e}}{10^{16}}\right) \\
+3.5 A\left(\frac{N_{e}}{10^{16}}\right)^{1 / 4} x\left[1-\frac{3}{4} N_{\mathrm{D}}^{-13}\right] \omega\left(\frac{N_{e}}{10^{16}}\right),
\end{gathered}
$$

where $A$ is the ion broadening parameter, $\omega$ is the Stark width parameter, and $N_{\mathrm{D}}$ is the number of particles in the Debye sphere. Both $\omega$ and $A$ are weak functions of temperature. The contribution is almost due to elec- 

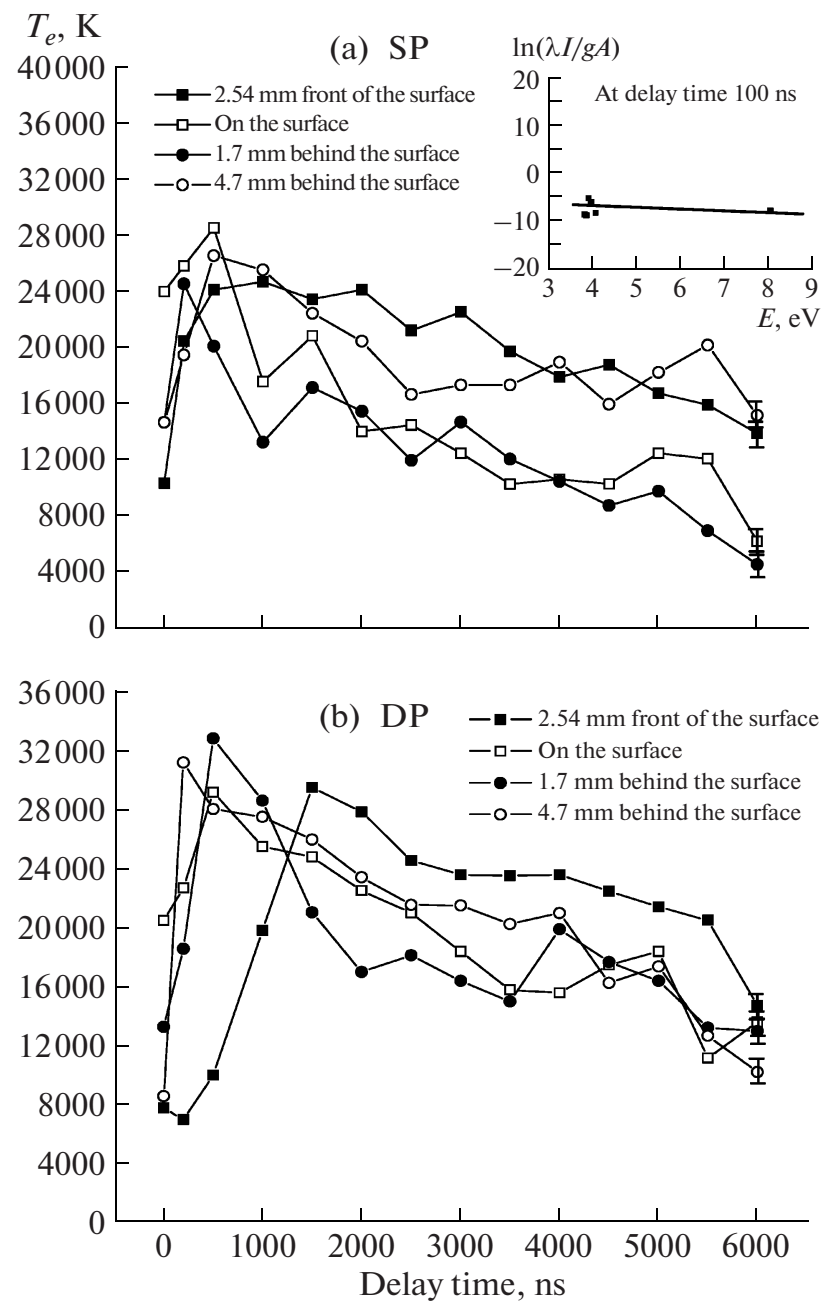

Fig. 8. (a, b) The electron temperature plotted versus delay time of Ti ionized lines at different laser focal positions relative to the target surface for SP-and DP-excitation modes.

tron impact, and the half-width of the Stark-broadened transition can be estimated to a good approximation [37]. Under these assumptions, Eq. (4) reduces to

$$
\Delta \lambda_{1 / 2}(A)=2 \omega\left(\frac{N_{e}}{10^{16}}\right),
$$

where $N_{e}$ is the electron density in $\mathrm{cm}^{-3}$. The observed line width were corrected by subtracting the contribution of the instrumental width by using the following relation

$$
\Delta \lambda_{\text {true }}=\Delta \lambda_{\text {observed }}-\Delta \lambda_{\text {instrument }} .
$$

It is worth mentioning that the plasma under study was assumed to be optically thin for the used lines and therefore it was unlikely to be subject to self-absorption. The line width was determined by numerical fitting of a Lorentzian profile. The measured electron
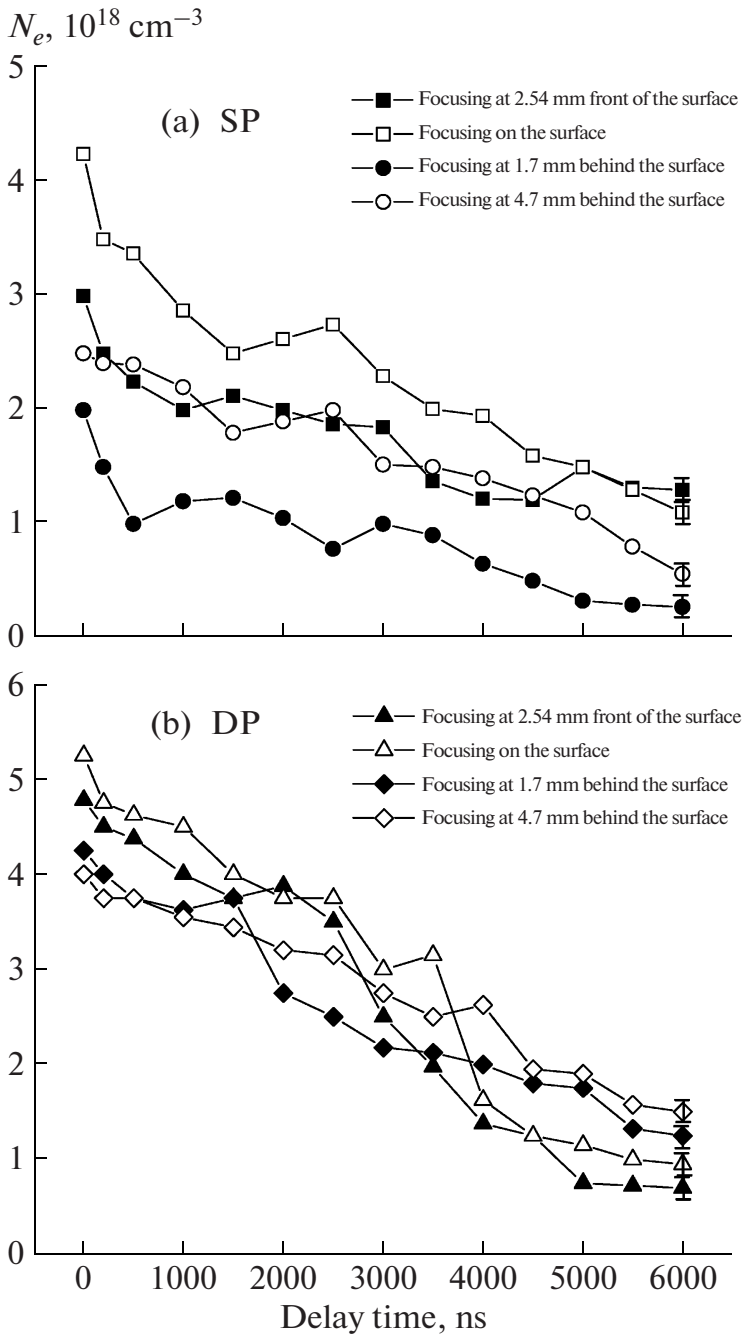

Fig. 9. (a, b) Electron density temporal evolution $N_{e}$ of Ti by focusing the laser beam at different laser focal positions relative to the target surface for $\mathrm{SP}$ - and DP-excitation modes.

densities for SP- and DP-modes of the Ti II $325.29 \mathrm{~nm}$ line at different positions are depicted in Fig. 9 as a function of delay time by focusing the laser beam on the target surface at ambient pressure. From 0 to $6000 \mathrm{~ns}$, the electron density decreases after plasma initiation and has values from $3,4.25,2,2.5 \times 10^{18} \mathrm{~cm}^{-3}$ to $1.3,1.1,0.27$ and $0.56 \times 10^{18} \mathrm{~cm}^{-3}$ for single pulse while $4.78,5.25,4.25,4 \times 10^{18} \mathrm{~cm}^{-3}$ to $0.7,0.95,1.25$, and $1.5 \times 10^{18} \mathrm{~cm}^{-3}$ for double pulse in different positions at $2.54 \mathrm{~mm}$ front of the surface, on the surface, $1.7 \mathrm{~mm}$ behind the surface, $4.7 \mathrm{~mm}$ behind the surface, respectively. It can be seen that the electron density in the case of DP-mode is about one order of magnitude higher than its values in the case of SP-modes.

The electron density profile reveals a near linear decay in electron density between 0 to $6000 \mathrm{~ns}$. This is somewhat in contrast to the non-linear changes in 
plasma transmission and plasma continuum emission during a similar temporal region, namely between 0 and $6000 \mathrm{~ns}$. The uncertainty associated with the assumed plasma temperature is greater in the region of more significant Stark broadening, however, even at the greatest electron number density measured the corresponding relative error is approximately $5 \%$.

In addition, we also observed saturation in the emission intensity for different ionic lines at higher laser irradiances. By increasing the laser irradiance, more excited species, free electrons and ions are produced which would have been interacted with the incoming laser photons and cause more heating and ionization which could increase the absorption of the more laser photons. It was found that the plasma life time for both SP and DP are slightly different, however, DP laser mode clearly produced high temperatures and dense plasma for the same amount of energy. Furthermore, the prompt electrons ejected from the $\mathrm{Ti}$ target collisionally excite and ionize ambient air molecules, which give enhancement in the emission intensity lines of Ti especially by using DP-mode. Also, it was seen that the electron density in the case of DP is a factor of about one order of magnitude higher than its values in the case of SP-mode.

\section{CONCLUSIONS}

LIBS technique was applied to study the temporal evolution of expanding laser-ablated Ti plasmas. This study revealed that the collinear DP-LIBS is superior as compared with SP-LIBS to understand the generation and expansion dynamics of the plasma induced by interaction of ns, Q-switched, Nd:YAG laser with Ti target. To the best of our knowledge, the experimental results obtained in the UV region from 312 to $334 \mathrm{~nm}$ for ionized $\mathrm{Ti}$ are being reported for the first time. It was noticed that the highest velocities of the plasma species with double laser pulses are in the direction normal to the target surface. Our Spectral analysis demonstrates the presence of highly ionized Ti lines emission. These lines are more intense in the case of DP than that in the SP-mode. The spectral intensity as a function of pulse delay time, laser irradiance and focal position of the laser beam with respect to the illumination surface was also investigated. It was observed that the intensity of Ti spectra is about five times for a DP-mode at $20 \mathrm{~mJ}$ as compared with a SP-mode for $40 \mathrm{~mJ}$ laser irradiance. In addition, the spatial studies carried out in this work, by focusing the laser at different distances, front, on and behind the surface, reveal that several Ti emission lines at Ti II (316.26), Ti II (321.71), Ti II (323.66), Ti II (325.29) and Ti II (332.29) $\mathrm{nm}$ for SP- and DP-modes in the UV regime are limited to within $6 \mathrm{~mm}$ behind the target surface at 0 delay between the laser pulse and triggering of ICCD camera at ambient pressure. The excitation temperature was about $28565,29163 \mathrm{~K}$ at $500 \mathrm{~ns}$ and the electron density was about $4.25 \times 10^{18} \mathrm{~cm}^{-3}, 5.25 \times$
$10^{18} \mathrm{~cm}^{-3}$ for SP- and DP-modes, respectively. This study can be useful for optimization conditions of a number of collinear DP-LIBS experiments to be employed in different applications.

\section{REFERENCES}

1. J. Roos, J. P. Celis, E. Vancoille, S. Boelens, F. Jungbult, J. Ebberink, and H. Homberg, Thin Solid Films 547, 193 (1990).

2. A. Chen, J. T. Scheure, C. Ritter, R.B. Alexander, and J. R. Conrad, J. Appl. Phys. 70, 6757 (1991).

3. J. Mizera, R. Y. Fillit, and T. Wierzchon, J. Mater. Sci. Lett. 17, 1291 (1998); K. T. Rie and T. H. Lampe, Mater. Sci. Eng. 69, 473 (1985).

4. A. De Giacomo, Spectrochim. Acta B 58 (1), 71 (2003).

5. A. De Giacomo, V. A. Shakhatov, and O. De Pascale, Spectrochim. Acta B 56, 71 (2001).

6. A. De Giacomo, M. Dell'Aglio, A. Santagata, and R. Teghil, Spectrochim. Acta B 60, 935 (2005).

7. M. Capitelli, A. Casavola, G. Colonna, and A. De Giacomo, Spectrochim. Acta B 59, 271 (2004).

8. Laser Induced Plasma and Applications, Ed. by L. J. Radziemski and D. A. Cremers (Marcel Dekker, New York, 1989).

9. M. Capitelli, F. Capitelli and A. Eletskii, Spectrochim. Acta B 55, 559 (2000).

10. S. Amoruso, Appl. Phys. A 69, 323 (1999).

11. S. Amoruso, R. Bruzzese, N. Spinelli and R. Velotta, J. Phys. B: At. Mol. Opt. Phys. 32, R131 (1991).

12. http://www.bigskylaser.com/brilliantseries.html.

13. http://www.roperscientific.de/datasheets/PurgedSP300. pdf.

14. NIST Atomic Spectra Database [http://www.nist.gov].

15. M. Borghesi, A. J. Mackinnon, R. Gaillard, O. Willi, and D. Riley, Phys. Rev. E 60, 7374 (1999).

16. M. A. Gondal and T. Hussain, Talanta 71, 73 (2007).

17. M. A. Gondal and T. Hussain, Energy Sources A 30, 441 (2008).

18. M. A. Gondal, T. Hussain, Z. H. Yamani, and M. A. Baig, Talanta 69, 1072 (2007).

19. V. Piñon, C. Fotakis, G. Nicolas, and D. Anglos, Spectrochim. Acta B 63, 1006 (2008).

20. Y. B. Zel'dovitch and Y. P. Raizer, Physics of Shock Waves and High-Temperature Hydrodynamic Phenomena (Academic, New York, 1966).

21. P. W. J. M. Boumans, "Excitation Phenomena and Temperature Measurements," in Theory of Spectrochemical Excitation, Ed. by P. W. J. M. Boumans (Hilger and Watts, London, 1966), Ch. 6, p. 92.

22. W. Lochte-Holtgreven, "Evaluation of Plasma Parameters," in Plasma Diagnostics, Ed. by W. Lochte-Holtgreven (Wiley Intersci., New York, 1968), Ch. 3, p. 135.

23. Principles of Laser Plasmas, Ed. by G. Bekefi (Wiley, New York, 1976), Ch. 13, p. 549.

24. A. A. I. Khalil, Surf. Coat. Technol. 200, 774 (2005). 
25. A. A. I. Khalil and N. Sreenivasan, Laser Phys. Lett. 2, 445 (2005).

26. A. A. I. Khalil and M. C. Richardson, Laser Phys. Lett. 3, 137 (2006).

27. H. Hora, Plasmas at High Temperature and Density (Springer, Heidelberg, 1991).

28. M. Borghesi, A. J. Mackinnon, R. Gaillard, O. Willi, and D. Riley, Phys. Rev. E 60, 7374 (1999).

29. H. R. Griem, Spectral Line Broadening by Plasmas (Academic, New York, 1974).

30. A. A. I. Khalil, M. Richardson, C. Barnett, and L. Johnson, J. Appl. Spectrosc. 73, 654 (2006).

31. C. Lopez-Moreno, S. Palanco, and J. J. Laserna, J. Anal. At. Spectrom. 20, 1275 (2005).
32. M. Corsi, G. Cristoforetti, M. Hidalgo, S. Legnaioli, V. Palleschi, A. Salvetti, E. Tognoni, and C. Vallebona, Appl. Geochem. 21, 748 (2006).

33. E. Tognoni, G. Cristoforetti, S. Legnaioli, V. Palleschi, A. Salvetti, M. Mueller, U. Panne, and I. Gornushkin, Spectrochim. Acta Part B 62, 1287 (2007).

34. S. Maurmann, V. A. Kadetov, A. A. I. Khalil, H.-J. Kunze, and U. Czarnetzki, J. Phys. D 37, 2677 (2004).

35. G. Cristoforetti, Spectrochim. Acta B 64, 26 (2009).

36. J. Ashkenazy, R. Kipper, and M. Caner, Phys. Rev. A 43, 5568 (1991).

37. H. R. Griem, Spectral Line Broadening by Plasmas (Academic, New York, 1974). 